\title{
Vortex lattice in a rotating holographic superfluid
}

\author{
Chuan-Yin Xia, ${ }^{1,2}$ Hua-Bi Zeng, ${ }^{1, *}$ Hai-Qing Zhang, ${ }^{3, \dagger}$ Zhang-Yu Nie, ${ }^{2}$ Yu Tian, ${ }^{4,1}$ and Xin $\mathrm{Li}^{4}$ \\ ${ }^{1}$ Center for Gravitation and Cosmology, College of Physical Science and Technology, \\ Yangzhou University, Yangzhou 225009, China \\ ${ }^{2}$ School of Science, Kunming University of Science and Technology, Kunming 650500, China \\ ${ }^{3}$ Center for Gravitational Physics, Department of Space Science \\ \& International Research Institute for Multidisciplinary Science, \\ Beihang University, Beijing 100191, China \\ ${ }^{4}$ School of Physical Sciences, University of Chinese Academy of Sciences, Beijing 100049, China \\ and Institute of Theoretical Physics, Chinese Academy of Sciences, Beijing 100190, China
}

\section{(Received 4 May 2019; published 9 September 2019)}

\begin{abstract}
By utilizing the AdS/CFT correspondence, we explore the dynamics of strongly coupled superfluid vortices in a disk with constant angular velocity at a finite temperature. Each vortex in the vortex lattice is quantized with vorticity $n=1$ by the direct inspection of their phases. As the angular velocity of the disk is greater than a critical value, the first vortex will be excited as expected from theoretical predictions. Subsequent vortices are also generated by increasing the rotation of the disk, resulting in remarkable step transitions of the angular velocity which excite each individual vortex. When the vortex number is large enough, the density of vortices is found to be linearly proportional to the angular velocity, which matches the Feynman relation very well. We also find that varying the temperature does not alter this Feynman relation.
\end{abstract}

DOI: 10.1103/PhysRevD.100.061901

\section{INTRODUCTION}

Quantized vortices have a profound effect on the behavior of type-II superconductors and superfluids. Quantized circulation is a macroscopic quantum-mechanical effect, which is a direct consequence of a single-valued wave function, where the phase must change by $2 \pi n$ (where $n \in Z$ is the vorticity) around a vortex core. Theoretical studies of equilibrium states predicted that the vortex lines would form a stable triangle lattice minimizing the free energy in both superconductors [1] and superfluids [2]. In experiments, vortex lines have been observed in both helium II in rotating containers [3-5] and Bose-Einstein condensation in cold atoms $[6,7]$. Vortex lattice formation and the vortex phase diagram under the constant rotation of a container can be simulated by numerically solving the powerful GrossPitaevskii (GP) equation [8-11]. However, the GP equation is only valid for a weakly coupled system at zero temperature, and studies of vortex formation in a strongly coupled superfluid at finite temperature are still lacking.

\footnotetext{
*hbzeng@yzu.edu.cn

"hqzhang@buaa.edu.cn
}

Published by the American Physical Society under the terms of the Creative Commons Attribution 4.0 International license. Further distribution of this work must maintain attribution to the author(s) and the published article's title, journal citation, and DOI. Funded by SCOAP .
The AdS/CFT correspondence [12-14] provides a complete description, "valid at all scales," of a strongly interacting quantum many-body system in terms of a classical gravitational system at finite temperature [15]. The holographic study of a superfluid (or superconductor) was originally introduced in Refs. [16-18], where the $U(1)$ gauge symmetry was spontaneously broken in an anti-de Sitter (AdS) planar black hole background. Later, the onevortex solution of this holographic model was obtained in both superfluids and superconductors in Refs. [19-22]. The triangle lattice solution of the vortex lattice was found from perturbative calculations near the critical point in Ref. [23]. The holographic superconductor/superfluid model also gives insights into the dynamics of a continuous phase transition even in far-from-equilibrium dynamics [24-28], the nonlinear response to a strong external field $[29,30]$, and the critical behavior of a nonequilibrium phase transition [31].

In this paper, we investigate the formation of vortex lattices in a strongly coupled holographic superfluid in a constantly rotating disk with angular velocity $\Omega$ when the temperature is away from zero. The quantized vortices are found by direct inspection of the phases of the scalar field. We also obtain the step transitions of the critical angular velocities $\Omega_{c}$, which excite each individual vortex as the vortex number is relatively small. As the vortex number is large enough, the density of the vortices is found to be linearly proportional to the angular velocity, which is consistent with the Feynman relation [32]. 


\section{HOLOGRAPHIC MODEL}

A simple action for a holographic superfluid consists of a complex scalar field $\Psi$ with mass $m$, minimally coupled to a $U(1)$ gauge field $A_{\mu}$ [16-18],

$$
S=\int d^{4} x \sqrt{-g}\left[-\frac{1}{4} F^{2}-|D \Psi|^{2}-m^{2}|\Psi|^{2}\right],
$$

where $F_{\mu \nu}=\partial_{\mu} A_{\nu}-\partial_{\nu} A_{\mu}$ and $D_{\mu}=\partial_{\mu}-i q A_{\mu}$, where $q$ is the charge. The theory can be defined in an $\mathrm{AdS}_{4}$ black hole background with Eddington-Finkelstein coordinates,

$$
d s^{2}=\frac{\ell^{2}}{z^{2}}\left(-f(z) d t^{2}-2 d t d z+d r^{2}+r^{2} d \theta^{2}\right),
$$

in which $\ell$ is the AdS radius, $z$ is the AdS radial coordinate of the bulk, and $f(z)=1-\left(z / z_{h}\right)^{3}$. Thus, $z=0$ is the AdS boundary and $z=z_{h}$ is the horizon; $r$ and $\theta$ are, respectively, the radial and angular coordinates of the dual $2+1$ dimensional boundary, which is a disk in our model. The Hawking temperature is $T=3 /\left(4 \pi z_{h}\right)$. For simplicity, the probe limit is adopted in this paper by assuming that the matter fields do not affect the gravitational fields. The black hole background (2) is static (rather than rotating), and thus the superfluid is rotating relative to the disk; equivalently, we can treat the superfluid as a static observer, and then the disk would appear to be rotating due to the fact that the superfluid has zero viscosity. Without loss of generality, we rescale $\ell=z_{h}=1$. Therefore, the equations of motion (EoMs) can be written as

$$
\left(-D^{2}+m^{2}\right) \Psi=0, \quad \partial_{\mu} F^{\mu \nu}=J^{\nu},
$$

where $J^{\mu}=i\left(\Psi^{*} D^{\mu} \Psi-\Psi D^{\mu} \Psi^{*}\right)$ is the bulk current. Proper boundary conditions should be imposed in order to solve the EoMs (3). For simplicity, the axial gauge $A_{z}=0$ is adopted, as in Ref. [18]. We impose regular boundary conditions on all of the physical solutions at the horizon of the black hole. Explicitly, we set $A_{t}=0$ at the horizon, as in Ref. [33]. Other fields at the horizon can be determined from the previous time steps in the time evolution schemes, rather than calculating them by hand. Near the boundary $z=0$, the general solutions take the asymptotic form

$$
\begin{array}{r}
A_{\nu}(t, z, r, \theta)=a_{\nu}(t, r, \theta)+b_{\nu}(t, r, \theta) z+\mathcal{O}\left(z^{2}\right), \\
\Psi(t, z, r, \theta)=\Psi_{1}(t, r, \theta) z+\Psi_{2}(t, r, \theta) z^{2}+\mathcal{O}\left(z^{3}\right) .
\end{array}
$$

From the AdS/CFT dictionary, the coefficients $a_{r, \theta}$ can be related to the superfluid velocity along the $r, \theta$ directions, while $b_{r, \theta}$ are the conjugate currents [19]. The coefficients $a_{t}$ and $b_{t}$ are interpreted as the chemical potential and charge density in the boundary field theory, respectively; $\Psi_{1}$ is the source term and $\Psi_{2}$ is the vacuum expectation value $\langle O\rangle$ of the dual scalar operator. In the superfluid phase we always impose $\Psi_{1} \equiv 0$ and $a_{t}=\mu>\mu_{c}$ on the $z=0$ boundary. In this paper we choose $m^{2}=-2$, and thus the critical chemical potential is $\mu_{c} \sim 4.06$. In order to study the formation of a superfluid vortex lattice in a rotating disk, we impose the angular boundary condition on the $z=0$ boundary as [34]

$$
a_{\theta}=\Omega r^{2},
$$

where $\Omega$ is the constant angular velocity of the disk. While in the rotating frame of reference, the field $a_{\theta}$ represents the relative velocity between the superfluid and the reference frame of the rotating disk. Therefore, $a_{\theta}$ is also the velocity of the superfluid as seen from the rotating disk. Thus, the most convenient way to introduce rotation to the holographic superfluid is to assume a static disk on the black hole boundary, while the superfluid rotates relative to the disk. Because the superfluid is incompressible the existence of inertial forces cannot introduce a superfluid velocity along the radial direction of the disk, and thus the physics of a rotating superfluid in a static disk is equal to the physics of a static superfluid in a rotating disk. One should note that the definition of $\Omega$ above has mass dimension +2 . Therefore, the physical angular velocity should be scaled by the chemical potential, which is the energy scale of this system. By doing this, one can get the correct physical angular velocity with mass dimension +1 and avoid the superluminal problems. We also impose $a_{r}=0$ at the boundary since we assume that no superfluid flows in the radial direction of the disk. In our model, the radius of the boundary disk is set as $r=R$. The Neumann boundary conditions are adopted both at $r=R$ and $r=0$, i.e., $\partial_{r} h_{i}=0$, where $h_{i}$ represents all of the fields except $a_{\theta}$. Please note that this Neumann boundary condition is imposed over the whole range of $z$. The periodic boundary conditions are used along the $\theta$ direction, and thus we use the Fourier decomposition in the $\theta$ direction for all of the fields. Chebyshev spectral methods are used in the $(z, r)$ direction. Time evolution is simulated by the fourth-order Runge-Kutta method. The initial configuration at $t=0$ is chosen to be a homogenous superfluid state without any rotation at a fixed temperature below $T_{c}$.

The free energy $F$ of the system can be computed from the renormalized on-shell action $S_{\text {ren }}$, i.e., $F=T S_{\text {ren. }}$. The renormalized on-shell action consists of two parts, viz., $S_{\text {ren. }}=S_{\text {o.s. }}+S_{\text {c.t. }}$, where $S_{\text {o.s. }}$ is the bare on-shell action obtained by subtracting the equations of motions from the action (1) and $S_{\text {c.t. }}$ is the counterterm that removes the divergence near the $z=0$ boundary. Explicitly, $S_{\text {c.t. }}=$ $-\left.\int d t d r d \theta \sqrt{-\gamma} \Psi * \Psi\right|_{z=0}$, which is computed near the $z=0$ boundary, and $\gamma$ is the determinant of the reduced metric on the boundary surface. Therefore, the final form of the renormalized on-shell action is (in the numerical computation we set $q=1$ ) 


$$
\begin{aligned}
S_{\text {ren. }}= & -\left.\frac{1}{2} \int d t d z d \theta\left[\frac{1}{r} A_{\theta} \partial_{r} A_{\theta}\right]\right|_{r=R}+\left.\frac{1}{2} \int d t d r d \theta\left[r\left(-a_{t} b_{t}+\frac{1}{r^{2}} a_{\theta} b_{\theta}+\Psi_{1}^{*} \Psi_{2}+\Psi_{2}^{*} \Psi_{1}\right)\right]\right|_{z=0} \\
& +\frac{i q}{2} \int d t d z d r d \theta\left[\frac{r}{z^{4}} A_{\mu}\left(\Psi^{*} \partial^{\mu} \Psi-\Psi \partial^{\mu} \Psi^{*}-2 i q A^{\mu}|\Psi|^{2}\right)\right] .
\end{aligned}
$$

\section{QUANTIZED VORTEX LATTICE}

According to Landau's two-fluid model of a superfluid [35], the normal components behave like ordinary liquids while the superfluid components move without dissipation. These two components can have different velocities: $v_{n}$ for the normal parts and $v_{s}$ for the superfluid parts. If the container (a two-dimensional disk in our case) rotates at a constant angular velocity $\Omega$, the normal component rotates similarly to a rigid body. This implies that the linear velocity $v_{n}=\Omega \times r$ and the curl $\nabla \times v_{n}=2 \Omega$, in which $r$ is the position vector with its origin at the vortex core. In contrast, the superfluid part remains stationary, i.e., $v_{s}=0$ at small $\Omega$, which is called the Landau state. However, a stationary liquid in a rotating container implies a higher free energy. Thus, as $\Omega$ increases to a critical value $\Omega_{c 1}$, the Landau state becomes unstable and prefers to enter a state with one vortex. As $\Omega$ increases to the second critical velocity $\Omega_{c 2}$ two quantized vortices appear, located symmetrically in the disk. Consequently, higher angular velocities will excite subsequent vortices, which will arrange themselves in the disk according to the minimum of the free energy.

The top row of Fig. 1 shows the development of vortex lattices (with six vortices) from $t=0$ to the final equilibrium state at $t=600$ for $R=5$ and $\Omega=0.42$, at the temperature
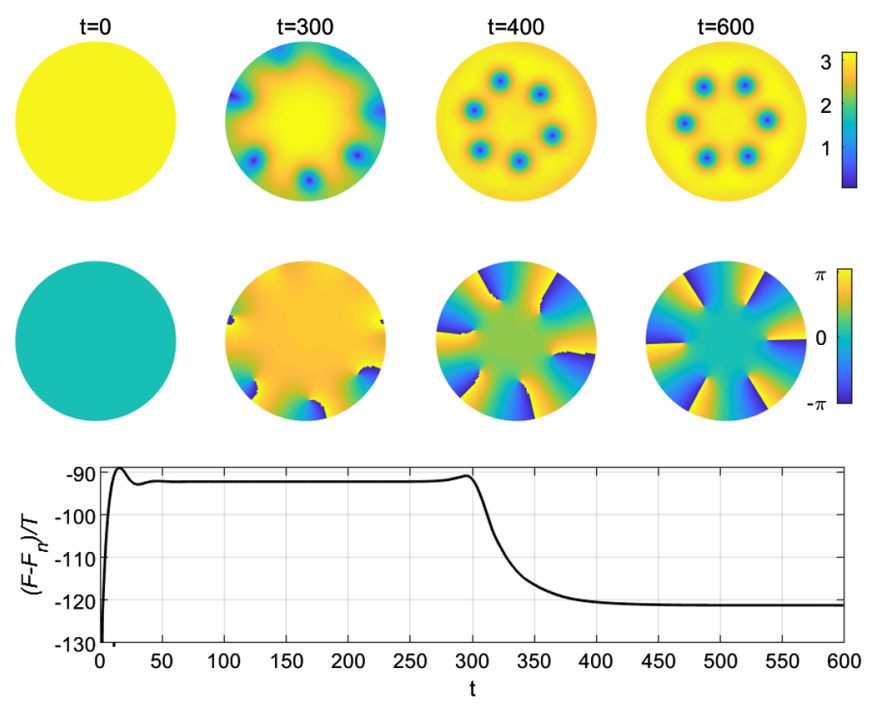

FIG. 1. Superfluid vortex lattice formation at separate times (top row), their corresponding phases (middle row), and the time evolution of the rescaled free energy (bottom row) for $R=5$ and $\Omega=0.42$. The temperature is chosen to be $T=0.82 T_{c}$.
$T=0.82 T_{c}$. At time $t \sim 300$ the vortices begin to form at the edge of the disk. This phenomenon is consistent with the theoretical studies and experiments in the literature [36]. At later times, vortices will rotate into the inner of the disk from the edge. The middle row of Fig. 1 shows the corresponding phases of the superfluids in the top row. In the final stable state, the locations of the vortices can be directly seen from the singularities or branch points of the phases. Circling around the vortex core, the phases vary from $-\pi$ (blue) to $+\pi$ (yellow) with discrepancy $2 \pi$, which demonstrates that each vortex is quantized with vorticity $n=1$. The bottom row of Fig. 1 shows the time evolution of the corresponding free energy $\left(F-F_{n}\right) / T$, in which $F_{n}$ is the free energy in the normal state, i.e., $\Psi=0$. One should note that the free energy formula is well defined in the equilibrium state, but may not be properly defined in the dynamical case. However, from Fig. 1 we see that at least at later times $t>400$ the system is in an equilibrium state with a relatively lower free energy, and in the regime $50<t<250$ the system is in a metastable state with higher free energy. Interestingly, we found a similar profile for the timedependent free energy in Ref. [37], where a superconductor under an inhomogeneous magnetic field was studied.

\section{CRITICAL ANGULAR VELOCITIES}

In Fig. 2, we show the typical stepwise relation between the number of vortices $N$ and angular velocity $\Omega$, from $N=1$ to $N=6$ for the case of $R=5$. The corresponding critical angular velocities $\Omega_{c 1}, \Omega_{c 2} \ldots$ can be read from the

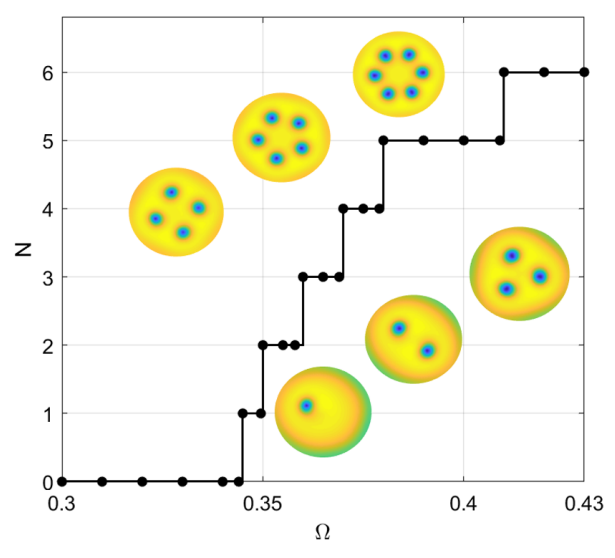

FIG. 2. Vortex number $N$ vs angular velocity $\Omega$ of the disk in the final stable state with $R=5$. The stepwise transitions signal the appearance of an additional vortex. The insets are the configurations of the stable vortices from $N=1$ to $N=6$. The temperature is $T=0.82 T_{c}$. 
jump of $N$. As the angular velocity increases from zero to $\Omega_{c 1} \sim 0.345$, the first vortex is excited by the rotation of the disk [35]. Subsequent vortices can also be generated at larger critical velocities. One possible reason for the unequal spacings is that the appearance of a vortex will in some sense break the superfluidity of the superfluid. The normal components of the superfluid will scatter at the vortices, leading to friction between the normal and superfluid components inside the disk [35]. This friction will also cost some energy, which may result in the unequal spacings of the critical angular velocities. Studies in condensed matter physics $[3,36,38]$ also showed the unequal spacings of the critical angular velocity as the vortex number is small.

\section{FEYNMAN RELATIONS}

For large vortex numbers, the rotation of the superfluid can be regarded as the rotation of a rigid body [35]. Therefore, from the path integral of the velocity along the disk circumference (enclosing all of the vortices) and the single-valued phase of the scalar field, one can readily get the Feynman relation [32] as

$$
N=\frac{M \Omega}{\pi} \pi R^{2}=n A,
$$

where $n \equiv \frac{M \Omega}{\pi}$ is the vortex number density, $M$ is the atomic mass of the superfluid, and $A \equiv \pi R^{2}$ is the area of the disk.

In Fig. 3 we show the configurations of vortex lattices with large vortex numbers for various values of $R, \Omega$, and temperature. From the Feynman relation (8), we can estimate the values of $M$ from the six panels in Fig. 3. We obtain $M(A) \sim 0.6122, M(B) \sim 0.8021, M(C) \sim 0.8390$, $M(D) \sim 0.8533, \quad M(E) \sim 0.8637$, and $M(F) \sim 0.7978$, respectively. The values of $M$ in panels $\mathrm{B}, \mathrm{C}, \mathrm{D}$, and $\mathrm{E}$ with more vortices are close since Eq. (8) is valid for a large number of vortices. In contrast, in panel A the vortex number
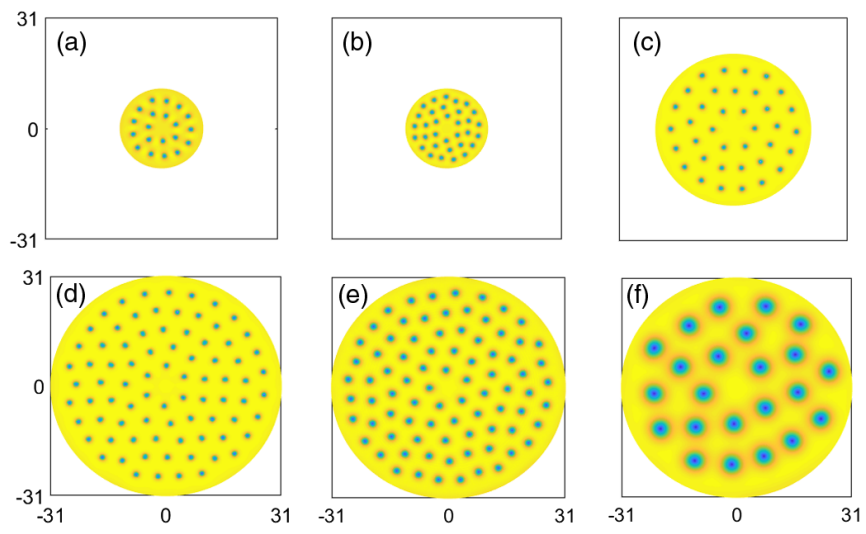

FIG. 3. Configurations of a large number of vortex lattices, with vortex number $N=20(A), 33(B), 37(C), 82(D), 83(E), 23(F)$, corresponding to $\left(R, \Omega, T / T_{c}\right)=(11,0.27,0.82), \quad(11,0.34$, $0.82), \quad(21,0.1,0.82),(31,0.1,0.82),(31,0.1,0.95),(31,0.03,0.98)$, respectively.
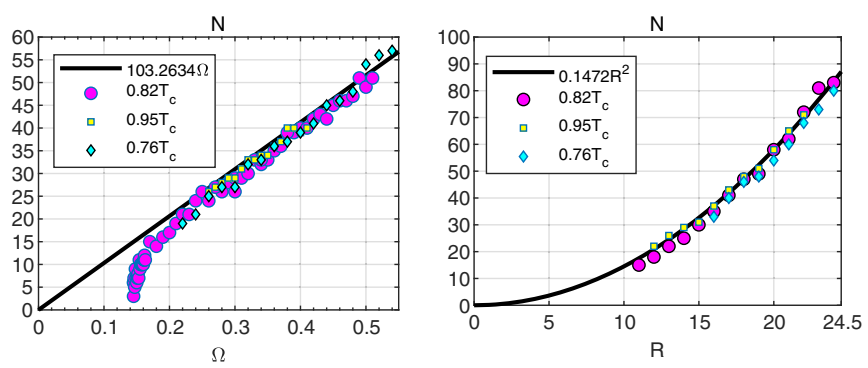

FIG. 4. Left: Vortex number $N$ vs angular velocity $\Omega$ for various temperatures with $R=11$. The black line $N \sim 103.2634 \Omega$ is the linear fitting curve as $N$ is large. Right: Vortex number $N$ vs the radius $R$ for various temperatures with $\Omega=0.17$. The black fitting curve is $N \sim 0.1472 R^{2}$.

$N=20$ seems to be not large enough to satisfy the Feynman relation (8). For this reason, we plot a large number of vortices with respect to $\Omega$ for various temperatures in the left panel of Fig. 4 by fixing $R=11$. One can readily see that because the vortex number is relatively large, the relations between $N$ and $\Omega$ for various temperatures are almost the same, which indicates that temperature does not alter the Feynman relation. For large vortex number, the linear relation between $N$ and $\Omega$ is fitted as $N \sim 103.2634 \Omega$. Thus, comparing this fitting line with Eq. (8), we can readily get that $M \sim 0.8534$. Therefore, the relation (8) becomes

$$
N \sim 0.8534 \Omega R^{2} .
$$

In the right panel of Fig. 4, we show the relation between the number of vortices and the radius $R$ for various temperatures by fixing the angular velocity $\Omega=0.17$. We can also see that different temperatures do not change this relation. The direct fitting of the curve in the right panel of Fig. 4 is $N \sim 0.1472 R^{2}$. By comparison, by using $\Omega=0.17$ in Eq. (9) we get $N \sim 0.1451 R^{2}$, which perfectly matches the fitting (within 1.43\%) from the right panel of Fig. 4. Therefore, this in turn numerically confirms the Feynman relation (8)!

\section{STATIC SINGLE-VORTEX SOLUTION}

We have investigated the dynamical evolution of the vortex lattices in the preceding sections. For completeness, in this section we study the static single-vortex solution in order to compare the first critical angular velocity $\Omega_{c 1}$ to that obtained from dynamics. Analytically, $\Omega_{c 1}$ can be obtained as [35]

$$
\Omega_{c 1}=\frac{1}{M R^{2}} \ln \left(\frac{R}{a}\right),
$$

where $a$ denotes the vortex core size. At the temperature $T=0.82 T_{c}$ and $R=11$, we find that $a \sim 0.6257$ by fitting the single-vortex order parameter as $\langle O(r)\rangle=$ $\langle O(R)\rangle \tanh (r /(\sqrt{2} a))$ [39], which is shown in the top 

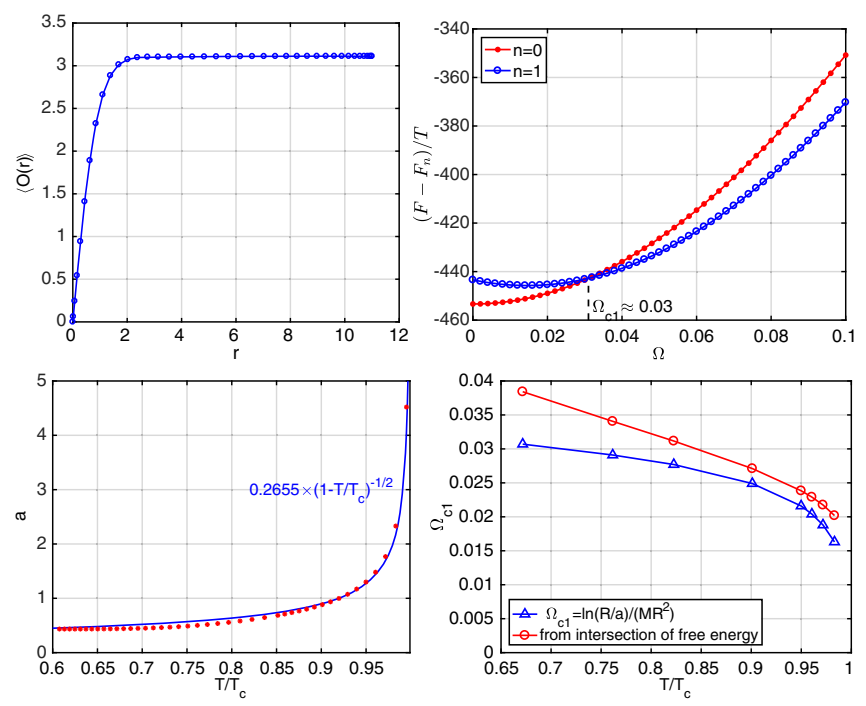

FIG. 5. Top left: Static single-vortex solution for $R=11$ at $T=0.82 T_{c}$. The vortex order parameter far from the vortex core is $\langle O(R)\rangle \sim 3.1114$. Top right: Free energy vs the angular velocity for the $n=0$ and $n=1$ vortex solutions in the static case with $R=11$ at $T=0.82 T_{c}$. Bottom left: The vortex size $a$ vs temperature. The blue line is the fitting curve. Bottom right: The first critical angular velocity vs temperature from two different approaches.

left panel of Fig. 5. Substituting $M \sim 0.8534$ from the above fitting (9) into Eq. (10), we get $\Omega_{c 1} \sim 0.0278$. By contrast, in the top right panel of Fig. 5 we show the free energies of the static $n=0$ and $n=1$ vortex solutions. The intersecting point $\Omega_{c 1} \sim 0.03$ is the first critical angular velocity, which is comparable to $\Omega_{c 1} \sim 0.0278$ (within $7.34 \%$ ) obtained from the analytical derivation (10). In the bottom panels of Fig. 5 we also show the temperature dependence of the vortex size $a$ and the first critical angular velocity $\Omega_{c 1}$. The time dependence of $a$ can also be vividly seen in panels D, E, and F in Fig. 3, where individual vortex grows as the temperature increases. The fitted curve of this relation is $a(T) \sim 0.2655 \times\left(1-T / T_{c}\right)^{-1 / 2}$, as shown in the bottom left panel of Fig. 5. In the bottom right panel of Fig. 5 we show the relation between $\Omega_{c 1}$ and temperature. The red curve and circles are from the intersections of the $n=0$ and $n=1$ free energies of static single-vortex solutions. The blue curve and triangles are obtained by substituting each $a(T)$ into Eq. (10). We see that the critical angular velocities obtained from these two different approaches are consistent with each other in the sense that as the temperature grows they will decrease, and their values are close to each other.

\section{SUMMARY}

Vortex lattices were found in a holographic superfluid at different temperatures for the first time. We also confirmed that the Feynman linear relation is always satisfied for different temperatures. This was the advantage of holography over the GP equation, which cannot deal with finitetemperature problems. The critical angular velocity from the analysis of the free energy also matched the theoretical predictions very well [Eq. (10)]. We expect that this holographic study may shed light on the properties of a rotating strongly coupled superfluid.

\section{ACKNOWLEDGMENTS}

We thank Christopher P. Herzog for valuable comments. This work is supported by the National Natural Science Foundation of China (under Grants No. 11675140, $11705005,11875095,11565017$, and 11675015). Y. T. is also supported by the "Strategic Priority Research Program of the Chinese Academy of Sciences" with Grant No. XDB23030000.

H. B. Z. and H. Q. Z. contributed equally to this work as corresponding authors.
[1] A. A. Abrikosov, Zh. Eksp. Teor. Fiz. 32, 1422 (1957) [Sov. Phys. JETP 5, 1174 (1957)].

[2] V. K. Tkachenko, Sov. Phys. JETP 22, 1282 (1966); Zh. Eksp. Teor. Fiz. 50, 1573 (1966) [Sov. Phys. JETP 23, 1049 (1966)]; 29, 945 (1969).

[3] R. E. Packard and T. M. Sanders, Jr, Phys. Rev. Lett. 22, 823 (1969).

[4] E. J. Yarmchuk, M. J. V. Gordon, and R. E. Packard, Phys. Rev. Lett. 43, 214 (1979).

[5] G. P. Bewley, D. P. Lathrop, and K. R. Sreenivasan, Nature (London) 441, 588 (2006).

[6] K. W. Madison, F. Chevy, W. Wohlleben, and J. Dalibard, Phys. Rev. Lett. 84, 806 (2000).
[7] J. R. Abo-Shaeer, C. Raman, J. M. Vogels, and W. Ketterle, Science 292, 476 (2001).

[8] C. J. Pethick and H. Smith, Bose-Einstein Condensation in Dilute Gases (Cambridge University Press, Cambridge, England, 2002).

[9] A. L. Fetter, Rev. Mod. Phys. 81, 647 (2009).

[10] K. Kasamatsu, M. Tsubota, and M. Ueda, Phys. Rev. Lett. 91, 150406 (2003).

[11] K. Kasamatsu and M. Tsubota, Phys. Rev. Lett. 97, 240404 (2006).

[12] J. M. Maldacena, Adv. Theor. Math. Phys. 2, 231 (1998).

[13] S. S. Gubser, I. R. Klebanov, and A. M. Polyakov, Phys. Lett. B 428, 105 (1998). 
[14] E. Witten, Adv. Theor. Math. Phys. 2, 253 (1998).

[15] A. Adams, P. M. Chesler, and H. Liu, Science 341, 368 (2013).

[16] S. S. Gubser, Phys. Rev. D 78, 065034 (2008).

[17] S. A. Hartnoll, C. P. Herzog, and G. T. Horowitz, Phys. Rev. Lett. 101, 031601 (2008).

[18] C. P. Herzog, P. K. Kovtun, and D. T. Son, Phys. Rev. D 79, 066002 (2009).

[19] M. Montull, A. Pomarol, and P. J. Silva, Phys. Rev. Lett. 103, 091601 (2009).

[20] V. Keranen, E. Keski-Vakkuri, S. Nowling, and K. Yogendran, Phys. Rev. D 81, 126012 (2010).

[21] O. J. C. Dias, G. T. Horowitz, N. Iqbal, and J. E. Santos, J. High Energy Phys. 04 (2014) 096.

[22] M. S. Wu, S. Y. Wu, and H. Q. Zhang, J. High Energy Phys. 05 (2016) 011.

[23] K. Maeda, M. Natsuume, and T. Okamura, Phys. Rev. D 81, 026002 (2010).

[24] K. Murata, S. Kinoshita, and N. Tanahashi, J. High Energy Phys. 07 (2010) 050.

[25] M. J. Bhaseen, J. P. Gauntlett, B. D. Simons, J. Sonner, and T. Wiseman, Phys. Rev. Lett. 110, 015301 (2013).

[26] W. J. Li, Y. Tian, and H. b. Zhang, J. High Energy Phys. 07 (2013) 030.
[27] X. Bai, B. H. Lee, L. Li, J. R. Sun, and H. Q. Zhang, J. High Energy Phys. 04 (2015) 066.

[28] X. Li, Y. Tian, and H. Zhang, arXiv:1904.05497.

[29] H. B. Zeng, Y. Tian, Z. Y. Fan, and C. M. Chen, Phys. Rev. D 93, 121901 (2016).

[30] H. B. Zeng, Y. Tian, Z. Y. Fan, and C. M. Chen, Phys. Rev. D 95, 046014 (2017).

[31] H. B. Zeng and H. Q. Zhang, Phys. Rev. D 98, 106024 (2018).

[32] R. P. Feynman, Prog. Low Temp. Phys. 1, 17 (1955).

[33] M. Natsuume and T. Okamura, Phys. Rev. D 95, 106009 (2017).

[34] O. Domenech, M. Montull, A. Pomarol, A. Salvio, and P. J. Silva, J. High Energy Phys. 08 (2010) 033.

[35] L. D. Landau and E. M. Lifshitz, Statistical Physics, Part 2 (Pergamon, Oxford, 1981).

[36] V. M. Ruutu, Ü. Parts, J. H. Koivuniemi, N. B. Kopnin, and M. Krusius, J. Low Temp. Phys. 107, 93 (1997).

[37] M. V. Milosevic and F. M. Peeters, Phys. Rev. Lett. 94, 227001 (2005).

[38] G. B. Hess, Phys. Rev. 161, 189 (1967).

[39] J.F. Annett, Superconductivity, Superfluids, and Condensates, Oxford Master Series in Condensed Matter Physics, 1st ed. (Oxford University Press, Oxford, 2004). 